\title{
Resonance-Assisted Tunneling in Near-Integrable Systems
}

\author{
Olivier Brodier, Peter Schlagheck, and Denis Ullmo \\ Laboratoire de Physique Théorique et Modèles Statistiques (LPTMS), 91405 Orsay Cedex, France
}

(Received 4 April 2001; published 19 July 2001)

\begin{abstract}
Dynamical tunneling between symmetry related invariant tori is studied in the near-integrable regime. Using the kicked Harper model as an illustration, we show that the exponential decay of the wave functions in the classically forbidden region is modified due to coupling processes that are mediated by classical resonances. This mechanism leads to a substantial deviation of the splitting between quasidegenerate eigenvalues from the purely exponential decrease with $1 / \hbar$ obtained for the integrable system. A simple semiclassical framework, which takes into account the effect of the resonance substructure on the invariant tori, allows one to quantitatively reproduce the behavior of the eigenvalue splittings.
\end{abstract}

DOI: $10.1103 /$ PhysRevLett.87.064101

Despite its genuinely quantal character, tunneling is strongly influenced, if not entirely governed, by the structure of the underlying classical phase space [1]. Changing the dynamics from integrable to chaotic substantially modifies the tunnel coupling between two wells, not only if the chaos affects the classical motion within the wells (as considered in [2]), but also if the destruction of invariant tori is entirely restricted to phase space domains far inside the classically forbidden region.

Consider, for instance, a classical system exhibiting, due to a discrete symmetry, two congruent but separate regular regions. Semiclassical Einstein-Brillouin-Keller quantization enables one to construct "quasimodes" within each of these regions (i.e., wave functions fulfilling the Schrödinger equation to all powers of $\hbar$ ), with exactly the same energy for any pair of symmetry related quantized tori. This degeneracy becomes eventually lifted by the nonclassical coupling between the quasimodes, which selects the symmetric and the antisymmetric linear combination of these states as "true" eigenstates of the quantum system.

This "dynamical tunneling" process [3] arises in both integrable and nonintegrable systems, but with substantially different effectiveness. Introducing an appreciable chaotic layer between the two regular regions significantly enhances the tunnel coupling as compared to the integrable case and induces a huge sensitivity of the coupling with respect to variations of external parameters [4-6]. This phenomenon was successfully explained by the interaction of the regular quasimodes with quantum states living semiclassically within the chaotic domain, which, due to their delocalized nature, assist at the connection between these quasimodes [5-7]. Based on this picture, random matrix descriptions of the chaotic part of phase space were shown to reproduce the statistical properties of the tunneling rates [6-9]. Recent wave chaos experiments on optical [10] and microwave cavities [11] confirm the relevance of chaos for the tunnel coupling in nonintegrable systems. For the classically forbidden component of the underlying coupling process, however, namely the continuation of the wave function from the quantized torus to the chaos border, a general semiclassical theory is still lacking.
PACS numbers: 05.45.Mt, 03.65.Sq, 03.65.Xp

In the present contribution, we shall not tackle this problem directly for strongly mixed dynamics, but consider a simpler situation, namely the nearly integrable regime for which chaos is not appreciably developed. Yet, we shall see that, despite a seemingly "regular" phase space, the tunnel decay of the wave function and the associated coupling rates are nontrivial and cannot be reproduced by an integrable approximation of the dynamics. To explain this, we shall emphasize the role of classical resonances which come into play through two important, but distinct aspects. The first one, the importance of which in this context has already been recognized by Bonci and co-workers [12], is the existence of level crossings induced by a resonant torus in the semiclassical spectrum. Alone, however, they do not induce any modifications, as they arise also in integrable systems. The second aspect concerns the coupling of near-degenerate levels, which is related to the influence of the destruction of resonant tori on the nearby phase space [13,14]. The main purpose of this Letter is to demonstrate that the combination of these two aspects form the basis of a mechanism which, via the exponentially decaying tail of the eigenstates, controls the tunneling between symmetric islands. At rather large values of $\hbar$, this process may imply only one resonance. Deeper in the semiclassical regime, however, several of them are generally involved.

To make things more concrete, we shall illustrate our discussion on a specific, one-dimensional time periodic, example, namely the kicked Harper [15]. Classically, this system is described by the Hamiltonian

$$
H=\cos p+\sum_{n=-\infty}^{\infty} \tau \delta(t-n \tau) \cos q,
$$

where $\tau$ represents the kick period as well as the perturbation strength. Figure 1(a1) shows the near-integrable classical phase space of the corresponding map for $\tau=1$ within the fundamental domain $-\pi \leq q, p \leq \pi$.

The quantization of the kicked Harper map is conveniently described by the propagator $U$ over one kick period, which is given by the product of the two unitary operators, $\quad U=\exp \left(-i \tau \hbar^{-1} \cos \hat{p}\right) \exp \left(-i \tau \hbar^{-1} \cos \hat{q}\right)$, 


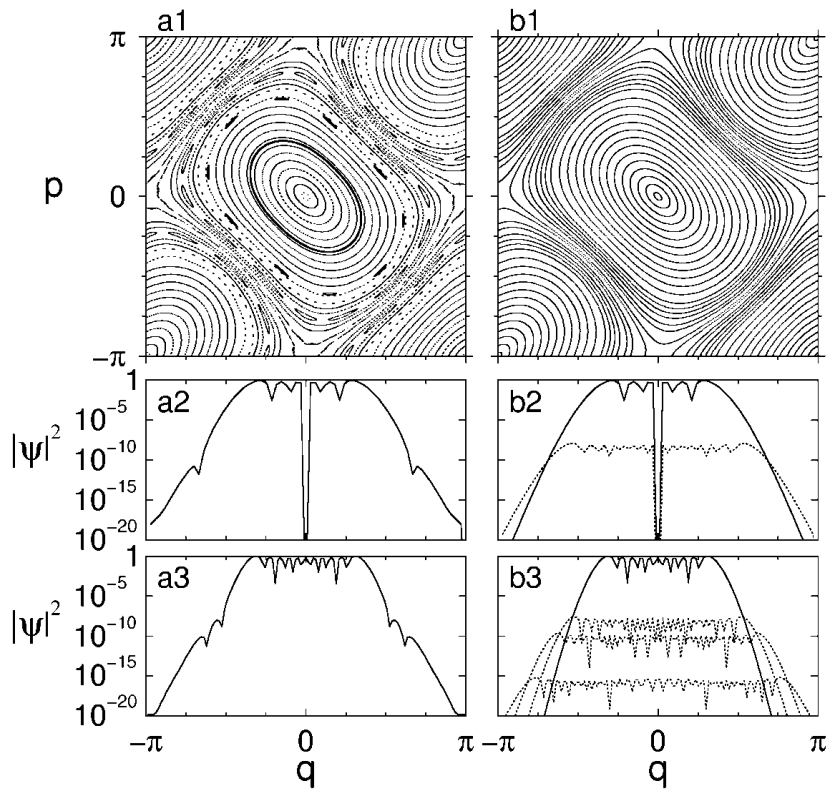

FIG. 1. (a1) Classical phase space of the kicked Harper at $\tau=1$. Boldly marked are the quantized torus with the action $I_{0}=\pi / 6$ as well as the island chain corresponding to the 10:1 resonance. (b1) Phase space generated by the integrable approximation $\tilde{H}$ [up to $\mathcal{O}\left(\tau^{6}\right)$ ] of the kicked Harper map. (a2),(a3) Probability densities of kicked Harper eigenfunctions at $\tau=1$ : (a2) 5 th excited state for $N=2 \pi / \hbar=66$; (a3) 10th excited state for $N=126$. (b2),(b3) Eigenfunctions of $\tilde{H}$ at $\tau=1$, weighted by their overlap with the kicked Harper eigenstate in (a2) and (a3), respectively: (b2) 5th (solid line) and 15th excited state (dotted line) for $N=66$; (b3) 10th (solid line), 26th (upper dotted line), 36th (middle dotted line), and 50 th excited state (lower dotted line) for $N=126$.

representing the kick and the free propagation between the kicks, respectively. For $\hbar=2 \pi / N$ with integer $N$, the $2 \pi$ periodicity in $q$ and $p$ permits writing the eigenfunctions $\psi$ as Bloch-like functions in both position and momentum-e.g., by imposing $\psi(q+2 \pi)= \pm \psi(q)$ and $\hat{\psi}(p+2 \pi)=\hat{\psi}(p)$ with $\hat{\psi}$ the Fourier transform of $\psi$. This effectively reduces the eigenvalue problem $U \psi=e^{i \varphi} \psi$ to the fundamental domain, yielding, for each particular choice of the periodicity conditions, a finite spectrum with $N$ discrete eigenphases $\varphi_{n}$.

Figure 1(a2) shows the probability density of a typical eigenfunction in the near-integrable regime at $\tau=1$, for $\hbar=2 \pi / N$ with $N=66$. The wave function corresponds to the 5th excited state within the central regular region around $(q, p)=(0,0)$ and is localized on the classical Kolmogorov-Arnol'd-Moser (KAM) torus with the action $I_{0}=\pi / 6$ (boldly marked in Fig. 1 ). We see that the eigenfunction exhibits the usual oscillatory structure within the torus and decreases exponentially beyond the caustics. However, this tunneling decay is not monotonous, but interrupted by a local "shoulder" at $q \simeq \pm 0.7 \pi$. For smaller $\hbar$ the same description applies except that several shoulders usually appear. See, e.g., the 10th excited state for $N=126$ in Fig. 1(a3).
The modification in the tunneling tail of the eigenfunctions has appreciable consequences for the eigenphase splitting $\Delta \varphi_{n}=\left|\varphi_{n}^{(+)}-\varphi_{n}^{(-)}\right|$between the "periodic" and the "antiperiodic" states [defined by $\psi(q+2 \pi)=$ $\pm \psi(q)]$, which is, in analogy to the energy difference between symmetric and antisymmetric states in double well problems, entirely dominated by the tunneling tails of the wave function. Figure 2 shows, as a function of $N=$ $2 \pi / \hbar$, the splittings for the $n$th excited states at $N=$ $6(2 n+1)$, which are all localized on the torus with action $I_{0}$. A clear departure from a purely exponential decay with $1 / \hbar$, to be expected for integrable dynamics, is observed.

In order to reveal the cause of this perturbation, we expand our wave function in terms of eigenstates of the time-independent Hamiltonian $\tilde{H}$ that is best adapted to the KAM structure of the regular region. This effective Hamiltonian can generally be expressed as an asymptotic series in the perturbation strength $\tau$. We use, in our case, a method introduced by Sokolov [16] and obtain

$$
\tilde{H}=\cos p+\cos q-\frac{\tau}{2} \sin p \sin q+O\left(\tau^{2}\right)
$$

in first order in $\tau$. Figure 1(b1) shows the phase space generated by the integrable approximation $\tilde{H}$ up to the 6th order in $\tau$. We see that the KAM tori of the kicked Harper are well reproduced within the regular region.

Calculating the overlap $\left|\left\langle\tilde{\psi}_{m} \mid \psi_{n}\right\rangle\right|$ between the kicked Harper eigenstate $\psi_{n}$ and the $m$ th excited eigenstate of $\tilde{H}$ identifies the structure in the tunneling tail in terms of contributions from the eigenstates of the integrable approximation. In fact, this overlap is, as expected, almost unity at $m=n$, decreases rapidly with $|m-n|$, and exhibits relatively pronounced local maxima for particular highly excited states of $\tilde{H}$. Plotting their probability

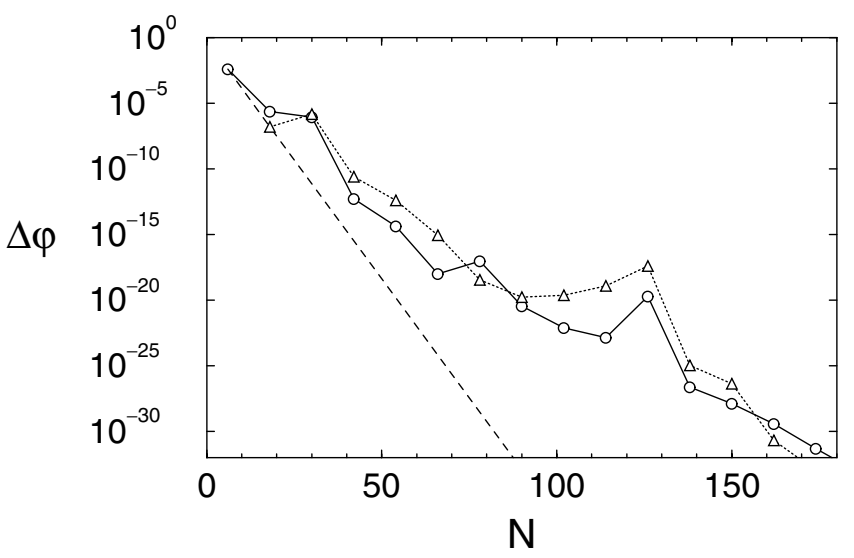

FIG. 2. As a function of $N=2 \pi / \hbar$, we plot the difference between the eigenphases of the periodic and the antiperiodic wave function [defined by $\psi(q+2 \pi)= \pm \psi(q)$ ] for the $n$th excited state at $N=6(2 n+1)$. Solid line: exact quantum result for the kicked Harper. Dashed line: eigenphase splittings of the corresponding states of the integrable approximation $\tilde{H}$. Dotted line: semiclassical result obtained by taking into account the effect of the $8: 1$, the $10: 1$, and the $14: 1$ resonance. 
densities [dotted lines in Figs. 1(b2) and 1(b3)] clearly confirms that it is the admixture of these particular components which modifies the tunneling decay of $\psi_{n}$.

This "selection rule" suggests a resonance phenomenon as underlying mechanism, principal aspects of which have already been discussed in the literature $[13,14]$. The classical phase space of the kicked Harper map exhibits island chains corresponding to nonlinear resonances between the kick and the unperturbed dynamics, two of which, the 10:1 and the 14:1 resonance, are visible in Fig. 1(a1). A $r: s$ resonance, with $r$ islands, generates in its neighborhood a periodic perturbation of $\tilde{H}$ and thereby couples $\tilde{\psi}_{n}$ to $\tilde{\psi}_{n+r k}$ with integer $k$, and it is precisely the combination of these couplings that gives rise to the shoulder structures in the tunneling tail.

To demonstrate that the above interpretation is basically correct, we use a semiclassical description of these couplings between quasistates to reconstruct the wave function in the tunneling regime and to reproduce the behavior of the fluctuations in the eigenphase splittings. Following the lines of the derivation undertaken by Ozorio de Almeida [14], we perform our semiclassical study in the action-angle variable representation $(I, \theta)$ associated with the integrable dynamics of $\tilde{H}$. In this representation, the kicked Harper Hamiltonian is written as $H=H_{0}(I)+$ $V(I, \theta, t)$ with $H_{0}(I) \equiv \tilde{H}(p, q)$ the unperturbed integrable Hamiltonian.

Let us, for the moment, assume that the phase space of the integrable system is perturbed by only one single island chain corresponding to a $r: s$ resonance. Following standard secular perturbation theory [17], we place ourselves in the corotating frame by the transformation $\theta \rightarrow \hat{\theta}=$ $\theta-2 \pi s t / r \tau$ to the slowly varying angle $\hat{\theta}$. It is then possible to perform a canonical transformation towards new action-angle variables $\left(I^{*}, \hat{\theta}^{*}\right)$ for which the perturbation $V$ is effectively replaced by its time-average $\bar{V}_{r: s}=$ $(1 / r \tau) \int_{0}^{r \tau} d t V\left[I^{*}, \hat{\theta}^{*}+2 \pi s t /(r \tau), t\right]$ over $r$ kick periods. This yields a time-independent Hamiltonian

$$
H_{r: s} \simeq H_{0}\left(I^{*}\right)-\frac{2 \pi s}{r \tau} I^{*}+\bar{V}_{r: s}\left(I^{*}, \hat{\theta}^{*}\right),
$$

for the effective dynamics in the vicinity of the $r$ :s resonance. The potential $\bar{V}_{r: s}$ is given by a $2 \pi / r$ periodic function of $\hat{\theta}$ and can therefore be written as

$$
\bar{V}_{r: s}=\sum_{k=1}^{\infty} V_{r: s}^{k} \cos \left(k r \hat{\theta}^{*}+\xi_{k}\right) .
$$

In practice, we directly extract the Fourier coefficients $V_{r: s}^{k}$, neglecting their action dependence, from the structure of the separatrix associated with the $r: s$ resonance. In the action-angle variable representation, the functional form $I_{\text {sep }}^{(+)}(\hat{\theta}), I_{\text {sep }}^{(-)}(\hat{\theta})$ of the "upper" and the "lower" separatrices between the resonance substructure and the KAM domain can be numerically constructed in a straightforward way. From the knowledge of $H_{0}(I)$ and from the condition that $H_{r: s}\left[I_{\text {sep }}^{( \pm)}(\hat{\theta}), \hat{\theta}\right]$ be independent of $\hat{\theta}$, the coefficients $V_{r: s}^{k}$ are calculated by the Fourier analysis of

$$
\bar{V}_{r: s}(\hat{\theta})+\left[H_{0}(I)-\frac{2 \pi s I}{r \tau}\right]_{I=I_{r: s}+\Delta I_{\text {sep }}(\hat{\theta})}=\mathrm{const}
$$

with $I_{r: s}$ the action at the $r: s$ resonance and $\Delta I_{\text {sep }}(\hat{\theta})=$ $\left[I_{\text {sep }}^{(+)}(\hat{\theta})-I_{\text {sep }}^{(-)}(\hat{\theta})\right] / 2$ [using $I_{r: s}+\Delta I_{\text {sep }}$ instead of $I_{\text {sep }}^{( \pm)}$, we compensate for the error that is introduced by the discrepancy between the $(I, \hat{\theta})$ and the $\left(I^{*}, \hat{\theta}^{*}\right)$ representation].

The kicked Harper eigenstates $\psi_{n}$ can now be explicitly constructed as linear combinations of the eigenstates $\tilde{\psi}_{n}$ of the integrable Hamiltonian within the framework of the first order perturbation theory, which involves the unperturbed eigenenergies $\tilde{E}_{n}$ of $\tilde{\psi}_{n}$ and the matrix elements $\left\langle\tilde{\psi}_{n^{\prime}}\left|\bar{V}_{r: s}\right| \tilde{\psi}_{n}\right\rangle$ of the perturbation $\bar{V}_{r: s}$. These matrix elements are directly calculated in the action-angle variable representation $(I, \hat{\theta})$; using Eq. (4) and representing the unperturbed eigenfunctions semiclassically as $\left\langle\hat{\theta} \mid \tilde{\psi}_{n}\right\rangle=\exp (i n \hat{\theta}) / \sqrt{2 \pi}$, we obtain $\left[\sigma=\operatorname{sgn}\left(n^{\prime}-n\right)\right]$

$$
\left\langle\tilde{\psi}_{n^{\prime}}\left|\bar{V}_{r: s}\right| \tilde{\psi}_{n}\right\rangle=\sum_{k=1}^{\infty} \frac{V_{r: s}^{k}}{2} \delta_{\left|n^{\prime}-n\right|, k r} \exp \left(i \sigma \xi_{k}\right) .
$$

We notice that nonzero couplings arise only between states the quantum numbers of which differ by integer multiples of $r$. As a consequence of this selection rule, the perturbative expression for the $n$th kicked Harper eigenstate involves only contributions $\tilde{\psi}_{n^{\prime}}$ with $n^{\prime}-n=k r$ for integer $k$, and the eigenphase splittings $\Delta \varphi_{n}=\left|\varphi_{n}^{(+)}-\varphi_{n}^{(-)}\right|$ between periodic and antiperiodic states are to a very good approximation given by

$$
\begin{aligned}
\Delta \varphi_{n} & =\sum_{k} \Gamma_{r: s}^{n, k} \Delta \tilde{\varphi}_{n+k r} \\
\text { with } \Gamma_{r: s}^{n, k \neq 0} & =\left|\frac{V_{r: s}^{k} / 2}{\tilde{E}_{n}-\tilde{E}_{n+k r}+2 \pi \hbar s k / \tau}\right|^{2}
\end{aligned}
$$

(and $\Gamma_{r: s}^{n, k=0} \equiv 1$ ). $\Delta \tilde{\varphi}_{n}=\left|\tilde{\varphi}_{n}^{(+)}-\tilde{\varphi}_{n}^{(-)}\right|$denotes the $n$th eigenphase splitting for the integrable Hamiltonian $\tilde{H}$ and can be semiclassically computed using standard WKB techniques for integrable dynamics. In principle, the sum on the right-hand side of (7) involves all couplings to the states the quantum numbers of which satisfy the selection rule $n^{\prime}-n=k r$. In practice, it is typically dominated by the contributions with quasienergies closest to degeneracy in the Floquet spectrum of the kicked system. Such a neardegeneracy occurs if the action of the $r$ :s resonant torus lies close to the arithmetic mean of the classical actions associated with $\tilde{\psi}_{n}$ and $\tilde{\psi}_{n+r k}$, in which case the energy difference $\tilde{E}_{n}-\tilde{E}_{n+k m}$ is determined by the linear approximation of $\tilde{H}$ near the resonant torus, which exhibits constant eigenphase spacing $2 \pi s / r$.

The dotted line in Fig. 2 shows the semiclassically calculated eigenphase splittings of the states $\psi_{n}$ that are localized on the torus with action $I_{0}$. Below $N=100$, only the 10:1 resonance is taken into account. Above $N=100$, 
multiple coupling schemes involving also the $8: 1$ and 14:1 resonances become important. This, however, does not pose any essential difficulty. Taking into account the effect of each $r$ :s resonance separately, we can recursively apply the mechanism described above for a single resonance, which amounts to generalize Eq. (7) to a multiple sum over products of $\Gamma_{r: s}^{n, k}$. This sum is again dominated by near-degenerate contributions; for $N=126$, for instance [Figs. 1(a3) and 1(b3)], it is given by $\Delta \varphi_{10} \simeq$ $\Gamma_{8: 1}^{10,2} \Gamma_{10: 1}^{16,1} \Gamma_{14: 1}^{36,1} \Delta \tilde{\varphi}_{50}$.

We see in Fig. 2 that the agreement with the exact quantum result is fairly good, though not perfect. Deviations from the quantum splittings occur due to neglecting the action dependence of the Fourier coefficients $V_{r: s}^{k}$ of the potential (4) and sometimes also due to accidental near degeneracies of $\psi_{n}$ with states that are associated with the "opposite" regular region centered around $(q, p)=$ $(\pi, \pi)$ beyond the separatrix. Nevertheless, the main features in the fluctuations of the level splittings are well reproduced by our semiclassical description.

In conclusion, we have given a simple prescription how to reproduce the tunneling rate fluctuations in the nearintegrable regime by the classical structure of a few important resonances. The effect of a $r: s$ resonance on the tunneling process of a regular eigenstate is twofold. On the one hand, the periodic modulation of the KAM torus structure due to the resonance induces appreciable couplings to higher states that are selected by the periodicity $r$ of the island chain. These couplings become, on the other hand, substantially enhanced at near degeneracies in the quasienergy spectrum. We have explicitly incorporated this mechanism in a perturbative semiclassical framework which, through simple iteration, is generalized to multiple coupling schemes involving several resonances. As an underlying picture, we obtain that each $r$ :s resonance allows the wave function to "hop" from one side of the island chain to the other, with the quantum number changing by integer multiples of $r$. For large $\hbar$ only one or a few resonances with relatively small $r$ come into play, whereas for small $\hbar$ the tunnel coupling proceeds via a succession of hops over several adjacent island chains, rather than by one single, but longer, classically forbidden event. Comparison with the quantum tunneling rates shows good agreement, and confirms in particular also that contributions from the chaotic part of phase space do not play a role in the nearintegrable regime.

Though exemplified only within the kicked Harper model, we expect that the resonance-assisted coupling phenomenon discussed here represents the fundamental tunneling mechanism for general near-integrable systems.
An open and interesting question is to which extent the role of the resonances retains its importance also in strongly mixed regular-chaotic systems where a macroscopic fraction of quantum states is associated with chaotic domains. We believe that at least the regular contribution to chaos-assisted tunneling, i.e., the coupling from the torus to the chaos border, is now amenable to straightforward reproduction via the identification of the major resonances inside the regular domain.

We thank E. Bogomoly, O. Bohigas, P. Leboeuf, S. Tomsovic, and A.M. Ozorio de Almeida for helpful and inspiring discussions. P.S. acknowledges financial support from Alexander von Humboldt-Stiftung and DFG. The LPTMS is an "Unité de recherche de l'Université Paris 11 associée au C.N.R.S."

[1] S. Creagh, in Tunneling in Complex Systems, edited by S. Tomsovic (World Scientific, Singapore, 1998), p. 1.

[2] S. C. Creagh and N. D. Whelan, Phys. Rev. Lett. 77, 4975 (1996); 82, 5237 (1999).

[3] M. J. Davis and E. J. Heller, J. Chem. Phys. 75, 246 (1981).

[4] W. A. Lin and L. E. Ballentine, Phys. Rev. Lett. 65, 2927 (1990); F. Grossmann, T. Dittrich, P. Jung, and P. Hänggi, Phys. Rev. Lett. 67, 516 (1991); R. Roncaglia et al., Phys. Rev. Lett. 73, 802 (1994).

[5] O. Bohigas, S. Tomsovic, and D. Ullmo, Phys. Rep. 223, 45 (1993); O. Bohigas, D. Boosé, R. Egydio de Carvalho, and V. Marvulle, Nucl. Phys. A560, 197 (1993).

[6] S. Tomsovic and D. Ullmo, Phys. Rev. E 50, 145 (1994).

[7] E. Doron and S. D. Frischat, Phys. Rev. Lett. 75, 3661 (1995); S. D. Frischat and E. Doron, Phys. Rev. E 57, 1421 (1998).

[8] F. Leyvraz and D. Ullmo, J. Phys. A 29, 2529 (1996).

[9] J. Zakrzewski, D. Delande, and A. Buchleitner, Phys. Rev. E 57, 1458 (1998).

[10] J. U. Nöckel and A. D. Stone, Nature (London) 385, 45 (1997)

[11] C. Dembowski et al., Phys. Rev. Lett. 84, 867 (2000).

[12] L. Bonci, A. Farusi, P. Grigolini, and R. Roncaglia, Phys. Rev. E 58, 5689 (1998).

[13] T. Uzer, D. W. Noid, and R. A. Marcus, J. Chem. Phys. 79, 4412 (1983).

[14] A. M. Ozorio de Almeida, J. Phys. Chem. 88, 6139 (1984).

[15] P. Leboeuf, J. Kurchan, M. Feingold, and D. P. Arovas, Phys. Rev. Lett. 65, 3076 (1990).

[16] V. V. Sokolov, Sov. J. Theor. Math. Phys. 67, 223 (1986); V. V. Sokolov, O. V. Zhirov, D. Alonso, and G. Casati, Phys. Rev. Lett. 84, 3566 (2000).

[17] A. J. Lichtenberg and M. A. Lieberman, Regular and Stochastic Motion (Springer-Verlag, New York, 1983). 